\title{
La utilización de los nuevos contextos digitales como una herramienta alternativa para la enseñanza de la Historia
}

Recibido: 16 de agosto de 2018 / Revisado: 11 de septiembre de 2018

Aceptado: 27 de noviembre de 2018 / Publicado: 19 de diciembre de 2018

\section{RESUMEN}

Esta investigación consiste en el análisis de los contenidos históricos de cinco blogs destinados a la enseñanza de las Ciencias Sociales que se encuentran en nuestro contexto digital. De entre todos los recursos que nos podemos encontrar en la web, hemos centrado nuestro análisis en los blogs como medio para la enseñanza y el aprendizaje. A través de ellos, hemos analizado su contenido sobre Historia y la manera en la que estos son presentados a los alumnos y también a cualquier usuario de la red. Con los resultados obtenidos, no solo hemos comprobado que los contenidos tratados se adecuan a las exigencias del currículum en el marco educativo, sino que también muchos docentes tienen a su alcance una nueva herramienta que, además de facilitar la enseñanza de la Historia, hace que resulte más atractiva a sus alumnos.

Palabras Clave: Blogs, Ciencias Sociales, Historia, Innovación en la enseñanza, Recursos Educativos.

\section{ABSTRACT}

The use of new digital contexts as an alternative tool for the teaching of History

This investigation consists in the analysis of the historical contents of five blogs destined to the teaching of the Social Sciences that are in our digital context. Of all the resources that we can find on the web, we have focused our analysis on blogs as a medium for teaching and learning. Through them, we have analyzed their content about History and the way in which these are presented to the students and also to any user of the network. With the results obtained, not only have we verified that the contents are adapted to the demands of the education curriculum, but also that many teachers have at their disposal a new tool that, in addition to facilitating the teaching of History, makes it more attractive to his students.

Keywords: Blogs, Educational Resources, History, Instructional Innovations, Social Sciences. 


\section{Introducción}

Una sociedad de la información como la actual, que se encuentra en constante evolución, ha promovido que haya cambios en el concepto de educación, haciendo que los docentes modifiquen su manera de educar y enseñar (Martín y Alonso, 2009). Debido a ello, nos encontramos con las TIC como una de las herramientas con más presencia en las aulas actuales, cuya rápida evolución ha hecho más accesible y fácil los procesos de enseñanza y aprendizaje (Molina, Valenciano y Valencia-Peris, 2015). El uso de ellas está permitiendo que la mayoría de los profesores comiencen a alejarse de las prácticas educativas tradicionales y opten por usar, a través de las TIC, nuevas más maneras de enseñar mediante los diferentes recursos que nos ofrecen.

Navegando por la red observamos las diferentes tipologías en las que se nos presentan los recursos educativos. En ella, nos encontramos videojuegos, fotografías, actividades y vídeos interactivos, documentales, programas educativos, blogs, páginas webs, entre otros muchos (Sobrino, 2013). De entre todas tipologías que se nos presentan en la web, nos llaman la atención los blogs, definidos por Checa (2014) como una herramienta que ha sido introducida en el ámbito educativo, ya que nos ofrece una gran cantidad de posibilidades en lo que respecta a la creación, participación y colaboración. Por tales motivos, consideramos que un blog es una herramienta útil que ayuda tanto a los maestros a innovar en sus técnicas de enseñanza como al alumnado a la hora de aprender nuevos conceptos, de la misma forma que constituyen un recurso positivo para reforzar aprendizajes previamente adquiridos. Analizando los blogs que se nos presentan en la web, destacamos que la gran mayoría han sido elaborados por docentes que los ponen en funcionamiento en sus clases. Por otro lado, observamos que se nos ofrecen contenidos de las diferentes áreas educativas recogidas por el currículum tanto de Educación Primaria como de Secundaria. Con ello, nos encontramos con blogs dedicados a la enseñanza de las matemáticas, lengua, ciencias sociales, etc.

De entre todos los blog que se nos presentan, nos centraremos en los que nos ofrecen contenidos relacionados con el área de Ciencias Sociales. Estos blogs nos muestran contenidos educativos referidos a Historia, Geografía y Arte, por lo que podemos considerarlos como una innovadora herramienta educativa ofrecida por las TIC, facilitando así que los maestros se alejen de la manera tradicional de impartir los contenidos relacionados con las Ciencias Sociales.

Desde el punto de vista del marco teórico, destacamos la aportación que nos hace Sobrino en relación a la evolución de las TIC, donde nos comenta que "el camino recorrido en el campo de las TIC durante los diez últimos años es asombroso, por cuanto que supone importantes rupturas con el tradicional proceso de enseñanza-aprendizaje" (Sobrino, 2009, p. 1). Esta evolución viene motivada debido a que "el uso de nuevas tecnologías favorece, además de poder desarrollar las propuestas de realidad aumenta- 
da, que se dé una comunicación fluida entre la comunidad de aprendizaje que conforman los diferentes grupos de trabajo" (Vicent e Ibáñez, 2012, p. 25). En coherencia con lo anterior, podemos señalar que una de las principales razones que han fomentado el auge de las TIC en las aulas es la facilidad de comunicación y uso. Junto con ello, el uso de las TIC en la enseñanza tiene una repercusión en la formación de los docentes, ya que como bien nos indica Domínguez (2012) deberán de tener conocimientos teóricoprácticos que les doten de la capacidad para usar y reconceptualizar los medios, pudiendo así fomentar de manera técnica, instrumental o práctica las diferentes tecnologías que vayan a usar en el aula. A pesar de la necesaria formación del profesorado en la utilización de los recursos tecnológicos, los resultados del estudio realizado por Almerich, Suárez-Rodríguez, Belloch y Bo (2012) nos muestran como el 72\% de los docentes se sienten inseguros en relación a la incorporación y utilización de las TIC en clase, a diferencia de un $8 \%$ que sí se ven capaces de integrarlas en las aulas.

Aun así, nos encontramos con un recurso TIC que cada vez más docentes están haciendo uso de él siendo este el blog. En función de lo comentado anteriormente, el blog es un recurso educativo que tiene como objetivo ser un apoyo en el proceso de enseñanza y aprendizaje en el ámbito educativo, haciendo que los maestros creen sus propios blogs donde comparten todo tipo de ideas y herramientas tanto para sus alumnos como para otros usuarios (Cuerva, 2007, cit. en Aznar y Soto, 2010). La utilización de este recurso ha supuesto una evolución en la enseñanza de los contenidos de las diferentes áreas, siendo una de las más beneficiadas las Ciencias Sociales donde autores como Sobrino nos argumentan que:

De un modo particular, de entre las aplicaciones de la Web 2.0 los blogs se han erigido como una herramienta de gran utilidad a la hora de almacenar recursos, actividades, comunicarse con los alumnos o implementar proyectos, existiendo en la actualidad un buen número de profesores de Geografía e Historia que trabajan con ellos a diario (Sobrino, 2013, p. 13).

En relación a ello, podemos mencionar que una de las principales razones que llevan a los profesionales de la enseñanza a usar los blogs es debido a las ventajas que este recurso educativo ofrece tanto en lo relacionado con la enseñanza como con el aprendizaje de los alumnos. Durán (2010, cit. en Sobrino, 2013) nos propone una serie de ventajas relacionadas con su utilización, destacando el fomento de la participación de los alumnos en la asignatura, la facilidad con la que los alumnos pueden expresar sus ideas y opiniones acerca de temas actuales y el acceso que tienen a nuevos contenidos relacionados con la materia así como a la solución de dudas y el desarrollo del pensamiento crítico. A pesar de todas estas ventajas, muchos docentes se encuentran reticentes a la hora de innovar en sus clases de Historia enfocando sus clases en el uso de técnicas tradicionales, con el uso predominante del libro de texto (Sánchez, González, Revilla y Martínez, 2015). 
A pesar de la reticencia de algunos docentes en innovar en sus prácticas educativas a través de los nuevos entornos digitales, autores como Tamayo y Ruiz (2016) hacen hincapié en la facilidad que tiene el uso de los blogs a la hora de que los estudiantes interioricen el contenido tratado, debido a que estos se presentan de una manera más atractiva para ellos ya sea mediante vídeos, mapas, etc. Por ello, se considera importante concienciar a los docentes en el uso de técnicas innovadoras en la enseñanza que nos son facilitadas por los entornos digitales cada vez más presentes en las aulas.

Por consiguiente, surge esta investigación con la que queremos comprobar si los contenidos que nos ofrecen los blogs son útiles para la enseñanza de las Ciencias Sociales, más específicamente los relacionados con la enseñanza de la Historia. Para ello, se ha realizado el análisis de los contenidos históricos de cinco blogs educativos de Educación Secundaria y Bachillerato de Ciencias Sociales. Los blogs que se han escogido son: Didáctica de las Ciencias Sociales y TIC, Claseshistoria.com, Tus Ciencias Sociales, Bitácora de clase. Ciencias Sociales, Geografía e Historia y Blog del profe Jaime. Uno de los motivos por los que hemos escogido estos blogs, ha sido su alto nivel de impacto en el área reflejado en su número de seguidores y visitas. Por otra parte, es importante mencionar que los blogs destinados a la enseñanza de la Educación Secundaria y Bachillerato tiene un mayor número de seguidores que los de Educación Primaria, siendo esta otra de las razones que nos ha llevado a centrarnos en los de Secundaria y Bachillerato. Con cada uno de los cinco blogs se realizará un análisis de su contenido, más específicamente el relacionado con la enseñanza de la Historia. Junto a ello, también, nos centraremos en la presencia que tienen los blogs en las redes sociales, como una forma de hacerse llegar a más usuarios y que esta herramienta no solo sirva para ser usada en clase, sino que cualquier usuario de la red pueda sacar partido de ella.

\section{Hipótesis y objetivos de la investigación}

En función de todo lo comentado anteriormente, la presente investigación pretende dar respuesta a las siguientes hipótesis:

- ¿Son válidos para la enseñanza y aprendizaje de la Historia los contenidos que nos presentan los blogs educativos?

- ¿Son adecuados los blogs educativos para ser utilizados como un recurso alternativo para la enseñanza?

Para poder dar respuesta a las hipótesis planteadas se han establecido una serie de objetivos generales:

1. Analizar el contenido de carácter histórico que nos presenta cada uno de los cinco blogs elegidos.

2. Comparar los distintos contenidos históricos que ofrecen los cinco blogs. 
A continuación, se plantean los objetivos específicos que nos permitirán alcanzar cada uno de los objetivos generales:

1.1 Evaluar el contenido del blog y su adecuación al curso al que va dirigido.

1.2 Comprobar la realidad que muestran los contenidos de los blogs relacionados con la Historia.

2.1 Catalogar cada uno de los blogs en función de sus contenidos.

2.2 Considerar la posibilidad de los nuevos entornos digitales como una nueva forma de generar conocimiento.

\section{Metodología}

Para poder cumplir con los objetivos establecidos y así dar respuesta a las hipótesis propuestas, la metodología empleada ha consistido en una revisión bibliográfica de una serie de publicaciones científicas relacionadas con la utilización del blog como una herramienta educativa y la enseñanza de las Ciencias Sociales, más concretamente de la Historia, permitiéndonos elaborar nuestro marco teórico y referencial.

Con lo que respecta al procedimiento llevado a cabo para seleccionar los blogs, se ha utilizado una metodología cualitativa consistente en un sistema predeterminado de categorías. Bisquerra (2014) nos argumenta que este sistema se basa en la búsqueda de temas comunes o agrupaciones conceptuales, siendo la categorización, donde se divide y simplifica el contenido acorde a criterios relacionados con el tema, y la codificación, donde se le aplica al contenido el código de la categoría asignada, dos de las operaciones que más caracterizan este sistema. Para nuestra investigación, se ha partido de categorías como "blog" y "blog educativo" y a partir de ellas, se han desarrollado otras subcategorías como son "blog de Ciencias Sociales" y "blog de Historia". Este procedimiento se ha realizado a través del buscador de Google.com, empleando las categorías y a continuación las subcategorías. Finalizado este proceso, nos han aparecido 334 blogs con similares características. Una vez realizada una lectura rápida de los mismos, nos hemos decido por la elección de los cinco ya mencionados blogs. Con los blogs ya seleccionados, hemos procedido al análisis de los contenidos sobre Historia. Este análisis se ha realizado mediante una tabla de seis entradas para cada blog. En cada una de las tablas se han recogido los contenidos históricos que nos ofrecen los blogs en cada uno de sus cursos $\left(1^{\circ}, 2^{\circ}, 3^{\circ}\right.$ y $4^{\circ}$ de E.S.O. y $1^{\circ}$ y $2^{\circ}$ de Bachillerato). Una vez recogidos, han sido categorizados en relación al recurso en el que se encuentran presentados, es decir, indicando si son textos explicativos, vídeos explicativos, documentales, mapas conceptuales, noticias, actividades, fotografías, enlaces, etc. (Ver tabla 1). Por último, una vez realizado este análisis se ha procedido a la comparación de los contenidos históricos de cada uno de los cinco blogs. 
Tabla 1. Fragmento de la tabla realizada para el análisis del blog Didáctica de las Ciencias Sociales y TIC.

\begin{tabular}{|c|c|}
\hline CURSO & CONTENIDO HISTÓRICO \\
\hline $1^{\circ}$ E.S.O. & 7. La prehistoria \\
\hline & $\begin{array}{l}\text { - Arqueología experimental y tecnología primate (vídeo demostrativo de cómo se vivía } \\
\text { en la Antigüedad) } \\
\text { - } \quad \text { Noticias (presencia de los neandertales y su forma de vida, cuevas, mandíbulas de ante- } \\
\text { pasados, especies que habitaron, tatuajes y arcos neolíticos) } \\
\text { - } \quad \text { Los primeros agricultores del Neolítico (documental) } \\
\text { - } \quad 20 \text { libros sobre Historia Antigua (recurso) } \\
\text { - La Edad de los metales (explicación, fotografías de monumentos y mapa conceptual) }\end{array}$ \\
\hline & 9. Primeras civilizaciones \\
\hline & - Momificación (explicación de cómo se realizaba, vídeo ello y documental) \\
\hline & 10. Grecia \\
\hline & $\begin{array}{l}\text { - } \quad \text { Troya (enlace donde se explica todo lo relacionado con la guerra) } \\
\text { - } \\
\text { - } \\
\text { - } \\
\text { - Petra, Esparta (vídeo inactivo) } \\
\text { Pueblos colonizadores (presentación) }\end{array}$ \\
\hline & 11. Roma \\
\hline & $\begin{array}{ll}\text { - } & \text { La historia de Roma (Podcast) } \\
\text { - } & \text { El mundo Romano y Prerromano con Astérix (enlace a cómics) } \\
\text { - } & \text { Noticias (monedas romanas) } \\
\text { - } & \text { Pompeya (vídeo cronológico sobre la tragedia ocurrida) } \\
\text { - } & \text { Los números romanos (enlace sobre curiosidades) } \\
\text { - } & \text { Numancia (webquest con actividades a evaluar y documental) }\end{array}$ \\
\hline & 12. Hispania romana y visigoda \\
\hline & $\begin{array}{ll}\text { - } & \text { Nombre de origen visigodos (enlaces con los nombres) } \\
\text { - } & \text { Organización política e institucional (mapas conceptuales) } \\
\text { - } & \text { Invasiones bárbaras (textos explicativos) } \\
\text { - } & \text { La Hispania visigoda (presentación explicativa) } \\
\text { - } & \text { Noticia (tumba celta) } \\
\text { - } & \text { Visigodos (blogs interesantes relacionados con el tema) } \\
\text { - } & \text { La conquista de Numancia (vídeo explicativo) } \\
\text { - } & \text { Pueblos Prerromanos, Hispania romana y visigoda (videos explicativos de repaso) }\end{array}$ \\
\hline
\end{tabular}


A continuación, se explicarán los dos capítulos por los que está compuesta esta investigación. El primer capítulo contiene tres apartados. El primero se basa en la presentación de los cinco blogs mediante una ficha técnica de cada uno de ellos. En las fichas técnicas se recogen datos como son el autor del blog, la dirección web, el número de seguidores, la frecuencia de publicación, entre otros aspectos. En el segundo apartado, se recogen los resultados de cada uno de los cinco análisis realizados. En él, se realizarán comentarios sobre los aspectos más llamativos que presentan cada blog en relación a los contenidos que nos ofrecen, como así de los recursos mediante los que se presentan. En el tercer y último apartado de este capítulo, se procederá a la comparación de los contenidos y recursos de los cinco blogs, destacando de esta manera las similitudes y diferencias de cada uno de ellos. Para finalizar, en el último capítulo, se establecerán una serie de conclusiones en relación al trabajo y también, se podrá dar respuesta a las hipótesis planteadas al comienzo de la investigación.

\section{Impacto educativo del empleo de los blogs de ciencias sociales en la enseñanza}

\subsection{Fichas técnicas}

Consideramos fundamental poder conocer de antemano cada uno de los blogs con los que vamos a trabajar antes de realizar el análisis de los mismos. Por ello, a través de la elaboración de las fichas técnicas podremos tener un conocimiento más amplio de los blogs como así de sus autores y de su presencia en las redes sociales.

Tabla 2. Didáctica de las Ciencias Sociales y TIC

\begin{tabular}{|c|l|}
\hline Autor & Diego Sobrino López \\
\hline Filiación & CEO La Sierra (Prádena, Segovia) \\
\hline Currículum vitae & $\begin{array}{l}\text { Diego Sobrino es licenciado en Historia del Arte (2003) y en Historia (2005) } \\
\text { y cuenta con un Máster Oficial en Ciencias Sociales para la investigación en } \\
\text { Educación (2013) por la Universidad de Valladolid. En la actualidad, es profe- } \\
\text { sor de Geografía e Historia en el CEO La Sierra (Prádena, Segovia), también } \\
\text { forma a profesores en organismos como son el Instituto Nacional de Tecno- } \\
\text { logías Educativas y de la Formación o el Centro de Recursos y Formación } \\
\text { del Profesorado el TIC, entre otros. Por otro lado, codirige la revista ÍBER. } \\
\text { Didácticas de las Ciencias Sociales, Geografía e Historia y forma parte del } \\
\text { Comité Científico de la revista Proyecto Clío. }\end{array}$ \\
\hline
\end{tabular}




\begin{tabular}{|c|l|}
\hline Biografía & $\begin{array}{l}\text { Sus líneas de investigación son la Didáctica de las Ciencias Sociales a tra- } \\
\text { vés de las nuevas tecnologías y la Didáctica de la Historia. Podemos destacar } \\
\text { publicaciones tales como Desarrollo de la competencia digital en Ciencias } \\
\text { Sociales. Experiencias en } 3^{\circ} \text { de ESO (2015),El trabajo con blogs en Ciencias } \\
\text { Sociales, Geografía e Historia (2013) o El blog en el aula de historia (2011), } \\
\text { entre otras muchas más. }\end{array}$ \\
\hline Nombre del Blog & Didáctica de las Ciencias Sociales y TIC \\
\hline Dirección web & https://diegosobrino.com/ \\
\hline Año de creación & 2007 \\
\hline Frecuencia de actualización & Entre una y dos veces al mes. \\
\hline Redes Sociales & $\begin{array}{l}\text { Podemos encontrar presencia de este blog en la red social de Twitter, siendo } \\
\text { el nombre de usuario “@ dsobrino” y en Facebook mediante "Diego Sobrino } \\
\text { López". }\end{array}$ \\
\hline Seguidores & $\begin{array}{l}\text { 13.453 en su blog, } 10.200 \text { en su cuenta de Twitter (fecha de consulta } 19 \text { de } \\
\text { julio de 2018) y } 2.950 \text { seguidores en Facebook (fecha de consulta } 26 \text { de abril } \\
\text { de 2018). }\end{array}$ \\
\hline &
\end{tabular}

Tabla 3. Claseshistoria.com

\begin{tabular}{|r|l|}
\hline Autor & Jorge Juan Lozano Cámara \\
\hline Filiación & Instituto Juan de la Cierva (Vélez-Málaga) \\
\hline Currículum vitae & $\begin{array}{l}\text { Jorge Juan Lozano es licenciado en Geografía e Historia en la especialidad } \\
\text { de Historia Contemporánea (1979) y en la especialidad de Historia Moderna } \\
\text { (1982) por la Universidad de Granada. En la actualidad, es profesor de Geo- } \\
\text { grafía e Historia en el instituto Juan de la Cierva en Vélez-Málaga. }\end{array}$ \\
\hline Biografía & No consta. \\
\hline Nombre del Blog & Claseshistoria.com \\
\hline Dirección web & http://www.claseshistoria.com/index.html \\
\hline Frecuencia de creación & 2004 \\
\hline Redes Sociales & $\begin{array}{l}\text { Podemos encontrar presencia de este blog en Facebook con el nombre de } \\
\text { usuario “Claseshistoria” y en Twitter con el usuario “@ claseshistoria” aunque } \\
\text { observamos que ambas redes sociales no están actualizadas desde 2012. }\end{array}$ \\
\hline Seguidores & $\begin{array}{l}\text { En su página de Facebook cuenta con 26.944 seguidores y 27.063 me gustas } \\
\text { en su perfil y en Twitter con 409 seguidores (fecha de consulta 19 de julio de } \\
\text { 2018). }\end{array}$ \\
\hline & \\
\hline
\end{tabular}


Tabla 4. Tus Ciencias Sociales

\begin{tabular}{|c|c|}
\hline Autor & Teodosio Serrano Murillo \\
\hline Filiación & Instituto Averroes (Córdoba) \\
\hline Currículum vitae & $\begin{array}{l}\text { En la actualidad es profesor de Ciencias Sociales, Geografía e Historia en el } \\
\text { instituto Averroes en Córdoba. }\end{array}$ \\
\hline Biografía & No consta. \\
\hline Nombre del Blog & Tus Ciencias Sociales \\
\hline Dirección web & http://tuscienciassociales-teodosio.blogspot.com.es/ \\
\hline Año de creación & 2010 \\
\hline Frecuencia de actualización & Entre dos y tres veces al mes. \\
\hline Redes Sociales & Cuenta con presencia en Twitter con el usuario “@teos123”. \\
\hline Seguidores & $\begin{array}{l}\text { En su blog cuenta con } 35 \text { seguidores pero es importante destacar que tiene } \\
\text { 1.377.287 de visitas en su blog. Por otro lado, en Twitter cuenta con } 109 \text { se- } \\
\text { guidores (fecha de consulta } 19 \text { de julio de 2018). }\end{array}$ \\
\hline
\end{tabular}

Tabla 5. Bitácora de clase. Ciencias Sociales, Geografía e Historia

\begin{tabular}{|c|c|}
\hline Autor & Manuel Pimienta Muñiz \\
\hline Filiación & Instituto San Fernando (Badajoz) \\
\hline Currículum vitae & $\begin{array}{l}\text { Es licenciado en Geografía e Historia con la especialidad en Geografía y tiene } \\
\text { un Máster en Gestión de Recursos Ambientales por la Universidad de Extre- } \\
\text { madura. Hasta el año 2003, impartió clases en el Departamento de Geografía } \\
\text { y Ordenación del Territorio de la misma universidad. En la actualidad trabaja } \\
\text { como profesor de Geografía e Historia en el instituto San Fernando de Badajoz. }\end{array}$ \\
\hline Biografía & $\begin{array}{l}\text { Entre sus publicaciones destacan artículos como La iniciativa comunitaria IN- } \\
\text { TERREG III en España (2005) y La Estrategia Territorial Europea (E.T.E.): } \\
\text { una concepción integradora y cohesionada del espacio (2003). Por otro lado, } \\
\text { también cuenta con colaboraciones en obras colectivas como son por ejemplo, } \\
\text { La experiencia de implantación de la sección bilingüe en el IES Extremadura } \\
\text { (Montijo-Badajoz): Consideraciones metodológicas y recursos en inglés para } \\
\text { la enseñanza de la Geografía (2011) y La iniciativa Interreg III para la coo- } \\
\text { peración de España y Portugal (2005). }\end{array}$ \\
\hline Nombre del Blog & Bitácora de clase. Ciencias Sociales, Geografía e Historia \\
\hline Dirección web & http://profe-manuel-pimienta.blogspot.com.es/ \\
\hline Año de creación & 2008 \\
\hline
\end{tabular}




\begin{tabular}{|c|l|}
\hline Frecuencia de actualización & Entre cuatro y cinco veces al mes. \\
\hline Redes Sociales & $\begin{array}{l}\text { Este blog tiene mucha presencia en las redes sociales, cuenta con Facebook } \\
\text { con el nombre "Profe Manuel Pimienta", Twitter con “@Prof_Manuel_P” e } \\
\text { Instagram con “@ profemanuelpimienta”. }\end{array}$ \\
\hline Seguidores & $\begin{array}{l}\text { Los seguidores de este blogs son 36 pero cuenta con } 863.639 \text { visitas, en Ins- } \\
\text { tagram cuenta con 75 seguidores y en Twitter con } 250 \text { seguidores (fecha de } \\
\text { consulta 19 de julio 2018). }\end{array}$ \\
\hline
\end{tabular}

Tabla 6. Blog del profe Jaime

\begin{tabular}{|c|l|}
\hline Autor & Jaime Castillo Sainz \\
\hline Filiación & Colegio Abad Sola (Gandía, Valencia) \\
\hline Currículum vitae & Actualmente es profesor en el Colegio Abad Sola de Gandía (Valencia). \\
\hline Biografía & $\begin{array}{l}\text { Entre sus publicaciones destacan artículos como Els feudals i la introducció } \\
\text { de la canyamel a la Safor del segle XV (1999) y Nueva toponimia para las } \\
\text { calles chamberileras (1996). También cuenta con colaboraciones en co- } \\
\text { lectivas como es El poder y la miseria: Leyes de pobres y prácticas represivas } \\
\text { en la Valencia bajomedieval (1996) y con libros como Alfons el Vell, duc reial } \\
\text { de Gandia (1999) y Els conflictes de l'aigua a la Safor medieval (1997). }\end{array}$ \\
\hline Nombre del Blog & Blog del profe Jaime \\
\hline Dirección web & http://jaimecast.blogspot.com.es/ \\
\hline Año de creación & 2010 \\
\hline Seguidores & $\begin{array}{l}\text { Cuenta con 51 seguidores y 1.990.499 visitas (fecha de consulta 19 de julio } \\
\text { de 2018). }\end{array}$ \\
\hline Erecuencia de actualización & Entre dos y tres veces al mes. \\
\hline No constan. \\
\hline Reciales
\end{tabular}

\subsection{Análisis de los cinco blogs}

\section{- Didáctica de las Ciencias Sociales y TIC}

Realizado el análisis de los contenidos históricos del blog, nos encontramos con 286 entradas repartidas entre los cursos de $1^{\circ}, 2^{\circ}$ y $4^{\circ}$ de E.S.O. y $2^{\circ}$ de Bachillerato. Con ello, observamos que el curso con más contenidos históricos es $2^{\circ}$ de Bachillerato seguido de 4 de la E.S.O. En lo referido al contenido que se nos ofrece, vemos que se presentan en orden cronológico, desde la prehistoria hasta el siglo XXI, por lo que el contenido no se repite en ningún curso de la E.S.O. Los contenidos que se presentan 
en $1^{\circ}$ se encuentran narrados de una manera más breve, centrándose en aspectos tales como son los pueblos romanos o visigodos, a diferencia de lo que se ofrece a partir de $2^{\circ}$ de E.S.O. donde vemos que se empiezan a centrar más en los personajes característicos de cada periodo como, por ejemplo, El Cid en la Edad Media o en hechos importante como son el Descubrimiento de América o la batalla de las Navas de Tolosa, entre otros más. A partir de $4^{\circ}$, observamos cómo aumentan las entradas dedicadas a Historia, ofreciéndose una gran cantidad de recursos destinados a explicar los contenidos y centrándose en muchos más aspectos como pueden ser el movimiento obrero, el crack del 29 o el fascismo, entre otros. En cambio, a partir de $2^{\circ}$ de Bachillerato vemos que algunos contenidos se repiten siendo estos los relacionados con la época romana, la Edad Media con temas como el Al-Ándalus, los reinos cristianos, el Antiguo Régimen, la Guerra Civil o el Franquismo entre otros. Cabe destacar que en este caso, los contenidos ofrecidos en este curso se centran más en la historia de España, por lo que el enfoque es distinto al que se les da en la E.S.O. Para finalizar, destacamos la aparición de contenidos que han ocurrido recientemente y que contribuyen a narrar hechos que están marcando y que marcarán la historia. (Fig. 1)

En lo referido a la manera en la que se presentan los contenidos, observamos que destacan los vídeos explicativos y documentales sobre el tema a tratar. También destacan los enlaces a páginas webs, artículos podcast, programas de radio y a recursos donde se explica el contenido más detalladamente como así recursos interactivos como flipped y webquest para trabajar contenidos de la Edad Media. Por otro lado, nos encontramos con entradas donde se nos presentan noticias como, por ejemplo, el Desastre de Annual y curiosidades sobre algunos contenidos históricos sirviendo de apoyo al tema. Asimismo, nos encontramos en menor medida con mapas políticos explicativos y presentaciones. Además de haber, como se ha comentado anteriormente vídeos explicativos, observamos que aparecen fragmentos de películas que narran algunos de los contenidos explicados en el tema siendo el caso de Los fusilamientos del 3 de mayo. En lo relacionado con el trabajo que realizan los alumnos, aparecen algunas entradas con fotos de los trabajos que ha elaborado el alumnado en clase. Además nos encontramos, en menor presencia con actividades que consisten en la resolución de cuestiones, realización de comentarios de texto, completar tablas y proyectos de investigación o debates (Fig. 2). Por último, es importante mencionar que muchos de los enlaces, vídeos explicativos y documentales se encuentran inactivos, por lo que no pueden ser utilizados.

\section{- Claseshistoria.com}

Una vez realizado el análisis de este blog, observamos que hay 297 entradas relacionadas con contenidos históricos, repartidos entre los cursos de $1^{\circ}$ y $4^{\circ}$ de E.S.O. y $1^{\circ}$ y $2^{\circ}$ de Bachillerato, encontrándose la mayoría en el curso de $4^{\circ}$ de E.S.O. Al igual que el anterior blog, los contenidos se nos ofrecen de manera cronológica, sin embargo, nos encontramos con la ausencia de algunos contenidos, debido a que no hay entradas para el curso de $2^{\circ}$ de E.S.O. Así, nos encontramos con contenidos sobre la Prehistoria, 
Egipto, Grecia, el Antiguo Régimen, los fascismos, la Primera y Segunda Guerra Mundial llegando a profundizar en aspectos de cada uno de ellos, como es la evolución del ser humano, la vida en el Paleolítico, la sociedad egipcia, las causas y consecuencias de las guerras, el movimiento obrero, etc. (Fig. 3) Por otra parte, el contenido de Bachillerato es escaso y nos encontramos con entradas dedicas a la colonización de América.

En relación a la representación de los contenidos, este blog se caracteriza porque se presentan de la misma manera, es decir, nos encontramos siempre con textos explicativos de cada una de las entradas. En ellos, suelen aparecer enlaces a textos característicos de la época que hacen referencia al hecho que se explica en la entrada. Además, destacan los mapas conceptuales que resumen el tema y en ellos, se encuentran enlaces directos a las diferentes entradas del tema. En menor frecuencia, se presentan fotografías de los hechos narrados y de alguno de los personajes más importantes de la etapa que se esté explicando. Es destacable, la aparición de muchas imágenes sobre carteles propagandísticos teniendo como objetivo demostrarle a los alumnos la función de transmisión de mensajes a la sociedad. Para finalizar, aparecen ejercicios interactivos predominando los crucigramas, los emparejamientos y realización de test, lo que pensamos que resulta motivador y muy beneficioso para el alumnado.

\section{- Tus Ciencias Sociales}

Con el análisis del blog realizado, comprobamos que de entre todas sus entradas 254 están relacionadas con la enseñanza de la Historia para Secundaria y Bachillerato. Estas están repartidas entre los cursos de $1^{\circ}, 2^{\circ}$ y $4^{\circ}$ de E.S.O. y $2^{\circ}$ de Bachillerato, siendo los dos últimos donde más presencia de contenidos hay. Los contenidos están presentados de manera cronológica, repartidos entre los tres cursos de la E.S.O. desde la Prehistoria hasta el final del siglo XX, por lo que hay contenidos sobre la Hominización y la evolución de los diferentes tipos del ser humano, las primeras civilizaciones, la antigua Grecia y su economía, sociedad y religión, el Imperio Bizantino, la Edad Moderna y todos los cambios que hubo, el absolutismo, la I Guerra Mundial, entre otros muchos contenidos. Asimismo vemos que no solo se limita a explicar los hechos, sino que profundiza en todos ellos, narrándonos muchas de las características que conciernan la época en cuestión. Por otra parte, los contenidos de $2^{\circ}$ de Bachillerato también se narran cronológicamente desde la Prehistoria hasta el año 2016 pero centrados en la narración de la historia española. Un aspecto característico de este blog es que contiene entradas relacionadas con la actualidad española, es decir, trata temas sobre los gobiernos recientes de España. A su vez, también se centra en muchos de los personajes históricos que han marcado la historia de España como por ejemplo Isabel II, los Reyes Católicos, Alfonso XIII o Amadeo I de Saboya entre otros. (Fig. 4)

En relación a los recursos que se nos presentan, observamos que al igual que el anterior, el contenido se presenta a través de textos explicativos aunque destacan los mapas políticos donde se nos ejemplifican las evoluciones de los reinos o de las batallas como, por ejemplo, mapas sobre la evolución del Imperio 
español del siglo XVI. Siguiendo en la misma línea, nos encontramos con mapas conceptuales y biografías de algunos de los personajes más importantes así como con fotografías de hechos. Destaca el hecho de que en muchas de las entradas nos aparezcan recomendaciones de películas que narran los hechos que se tratan en ellas, como es el caso de películas como Apocalypto, Sufragistas o La guerra del opio. Junto a lo anterior, también se presentan una serie de actividades que los alumnos deben de realizar como son comentarios de textos, resolución de preguntas, definición de conceptos, resumen de textos dados, indicación de la veracidad o falsedad de las afirmaciones, entre otras actividades. En menor medida, también aparecen ejes cronológicos y vídeos explicativos aunque algunos de ellos se encuentran inactivos.

\section{- Bitácora de clase. Ciencias Sociales, Geografía e Historia}

Tras el análisis realizado al blog, disponemos de 394 entradas que contienen contenidos históricos relacionados con la enseñanza de la misma. Los contenidos están repartidos entre los cursos de $4^{\circ}$ de la E.S.O. y $2^{\circ}$ de Bachillerato. Destaca el hecho de que aparece un tema de repaso desde la Prehistoria hasta la Edad Moderna. Por otra parte, en el curso de $4^{\circ}$ se comienza con contenidos relacionados con el Antiguo Régimen, la Guerra de Sucesión, la Revolución Industrial y sus factores, el marxismo, el Imperialismo, la Revolución rusa, etc. Además se trabajan temas relacionados con la Historia de España como, por ejemplo, la primera Guerra Carlista, la de Independencia, las Cortes de Cádiz, entre otros. En lo que respecta a $2^{\circ}$ de Bachillerato, se trabajan contenidos desde la Prehistoria hasta el fin de la Dictadura Franquista. Aquí nos encontramos con entradas relacionadas con la evolución de la Historia de España hasta la actualidad por ello, nos encontramos ante contenidos que se refieren a la Hispania romana y su economía, los visigodos, la convivencia de las tres culturas, la sociedad en el Al-Ándalus, la Dinastía de los Austria, el Despotismo ilustrado, la Primera República, etc.

En cuanto a los recursos en los que los contenidos son presentados en el blog, destacamos los vídeos relacionados con la serie de dibujos animados Érase una vez... El hombre que sirven para ilustrar partes de la historia. Asimismo, también destacan los vídeos explicativos, los mapas conceptuales y los ejes cronológicos que sirven como referencia al contenido del tema que se esté tratando y como resumen (Fig. 5). En menor medida, también aparecen contenidos en forma de diagramas sobre todo para explicar la evolución de la población y la economía así como, las pirámides de clases y los mapas políticos que muestran la evolución de una zona o las batallas que ha habido. Por último, son destacables algunos ejemplos de películas y fotografías que hacen referencia a algún contenido histórico. Además, nos encontramos con árboles genealógicos para hacer referencia a la evolución de las monarquías.

\section{- Blog del profe Jaime}

Una vez realizado el análisis del último blog, observamos que se ofrecen 163 entradas relacionadas con contenidos sobre Historia. Los contenidos están repartidos entre los cursos destinados a Educación 
Secundaria $\left(1^{\circ}, 2^{\circ}\right.$ y $\left.4^{\circ}\right)$, siendo $4^{\circ}$ de la E.S.O. el curso donde se encuentran la mayoría de los contenidos. Al igual que en los demás blogs analizados, el contenido se presenta de manera cronológica, desde la Prehistoria hasta la Guerra Fría. De entre todos ellos, destacamos contenidos como el bipedismo, Mesopotamia, los jeroglíficos, la época oscura, la Edad Media, el Cid, la Ilustración, el esclavismo, el periodo de entreguerras, etc. También son destacables las entradas dedicadas a personajes importantes de cada época como son por ejemplo las relacionados con el Cid, Mussolini, Alfonso XIII, Leonardo Da Vinci, entre otros (Fig. 6).

En relación a los recursos que se ofrecen en este blog, destacan las presentaciones con explicaciones en forma de mapas conceptuales. También son abundantes los vídeos y dibujos explicativos como así, los fragmentos de películas que hacen referencia al contenido que se presenta en ese momento en las entradas. Algunas de las películas que se recomiendan son Germinal, La lista de Schindler o Las uvas de la ira. Además destacamos el uso de mapa políticos que ejemplifican la evolución de los estados, destacando la utilización de un mapa animado sobre la expansión de la Alemania nazi. Por otro lado, nos encontramos aunque en menor medida, recursos como enlaces a blogs, en especial al blog de claseshistoria.com, y páginas web como Wikipedia o específicas sobre algún tema. Asimismo, observamos que se presentan biografías de algunos personajes, documentales y fotografías narrando así algunos de los contenidos ofrecidos, siendo también destacable la visita virtual que se propone para el contenido sobre la Cueva de Lascaux. Para finalizar, nos encontramos con diversas tipologías de actividades como son comentarios de texto, realización de test preparatorios de examen y actividades más interactivas como son la realización de crucigramas, quizz, murales virtuales o trabajos de investigación como, por ejemplo, la realización de una investigación sobre qué pasó con el cuerpo de Mussolini.

\subsection{Análisis comparativo}

Mediante el análisis del contenido de los cinco blogs dedicados a la enseñanza de las Ciencias Sociales, más concretamente en los destinados a la enseñanza de la Historia, hemos observado la cantidad de similitudes y diferencias que aparecen en los blogs con respecto al contenido como a la manera de representarlo, es decir, los recursos que se presentan.

En referencia al recuento de las entradas históricas, observamos que el blog que cuenta con más entradas es el del profesor Manuel Pimienta con 394 entradas, seguido del blog llamado Claseshistoria. com con 297, tras estos le sigue el del profesor Diego Sobrino con 286 entradas, por último nos encontramos con Tus Ciencias Sociales con 254 y El blog del profe Jaime con 163. En relación a esta información, podemos ver que los blogs analizados presentan un número parecido de entradas con contenidos históricos, aunque nos encontremos con una amplia diferencia entre el primero y el último. 
Con lo que respecta al histórico ofrecido por cada blog, observamos que la mayoría de ellos coinciden a la hora de ofrecerlos. Como vemos, la mayoría se muestran en orden cronológico desde la Prehistoria hasta la actualidad. Debemos de tener en cuenta que muchos de los blogs no están destinados a la enseñanza de todos los cursos de la E.S.O. y Bachillerato, por ende nos encontramos con la ausencia de algunos contenidos. Un ejemplo de ello, es por ejemplo el blog Claseshistoria.com donde no nos encontramos con contenidos referidos a la Edad Media y Edad Moderna, ya que no ofrece contenidos para $2^{\circ}$ de E.S.O. Otro caso parecido es el del blog Bitácora de clase. Ciencias Sociales, Geografía e Historia donde los contenidos se reparten entre dos cursos pero en este caso, el profesor del blog ofrece un repaso breve desde la Prehistoria hasta la Edad Moderna. En cambio, El blog del profe Jaime solo nos ofrece los contenidos históricos recogidos para la enseñanza de la Educación Secundaria, por lo que se omiten muchos contenidos sobre Historia de España. Con todo ello, podemos afirmar que el blog del profesor Diego Sobrino es el único que está destinado a todos los cursos, por lo que trabaja el temario de manera completa, a diferencia de los demás que omiten algunos de los contenidos. Por otro lado, observamos que casi todos los blogs coinciden en muchos contenidos como son el Antiguo Régimen, la I y II Guerra Mundial, la Antigua Grecia, la Revolución Industrial, la Guerra Fría, entre otros. De esta manera, nos damos cuenta de que la mayoría de los contenidos similares que nos presentan los blogs son los destinados a $4^{\circ}$ de E.S.O. aunque en menor medida, también coinciden otros pero no ya en todos los blogs. Siguiendo esta línea nos encontramos con blogs en los que se trabajan de manera más profunda contenidos, es el caso de los blogs Tus Ciencias Sociales y El blog del profe Jaime en los que a diferencia de los demás, trabajan de manera más intensa contenidos relacionados con la evolución del ser humano, la hominización o las etapas de la Grecia clásica. También destaca, por otra parte, el blog Tus Ciencias Sociales debido al hecho de que nos proporciona contenidos relacionados con los gobiernos democráticos más recientes como son los referidos al de Zapatero y al actual de Rajoy o el blog Didáctica de las Ciencias Sociales y TIC que nos ofrece noticias sobre hechos ocurridos recientemente como son los atentados de París 2015.

Con respecto a los recursos que se presentan, también observamos muchas diferencias y similitudes. A través del análisis realizado, hemos podido comprobar que el blog con más recursos es Didáctica de las Ciencias Sociales y TIC, porque nos presenta los contenidos mediante vídeos, documentales, noticias, podcasts, enlaces a otras páginas web y a fragmentos de programas de radio, recursos como flipped y webquest, fragmentos de películas, entre otros, encontrándonos así con una gran variedad de recursos para trabajar la Historia desde la innovación. Otros blogs que nos muestran unas características muy similares a este aunque no con tantos recursos son El blog del profe Jaime y Bitácora de clase. Ciencias Sociales, Geografía e Historia, donde nos ofrecen capítulos de los dibujos animados Érase una vez... El hombre, haciendo referencia a algún contenido histórico. Por otro lado, también nos presentan ejes cronológicos y presentaciones utilizadas en clase como así actividades sobre reflexionar sobre el visionado de películas, crucigramas, emparejar conceptos, etc. Como vemos, estos tres blogs presentan 
contenidos muy parecidos destacando estos por su carácter interactivo e innovador que los que puede ofrecer un libro, ya que mediante ellos se fomenta el uso de las TIC. Cabe destacar, que algunos de los contenidos que se ofrecen, en especial, algunos vídeos y enlaces se encuentran inactivos por lo que es imposible su uso. A diferencia de estos tres blogs donde se nos presentan una gran variedad de recursos, nos encontramos con Claseshistoria.com y Tus Ciencias Sociales que no nos presentan muchos recursos, en ellos predominan grandes textos explicativos y fotografías que hacen referencia al contenido. Por otra parte, Tus Ciencias Sociales destaca con respecto a los demás blogs por ofrecernos diversas actividades relacionadas con comentarios de textos, responder preguntas, indicar verdadero y falso o las causas y consecuencias de un hecho entre otras actividades, cosa que los demás blogs no presentan en cada tema sino que se encuentran repartidas a lo largo del blog. Es importante comentar el hecho de que casi todos los blogs coinciden en la utilización de películas como un recurso educativo, ya que hemos comprobado en muchos casos que se nos hacen referencias de películas para visualizar o se nos muestran algunos de los fragmentos de las mismas, sin embargo también se hacen referencia a series de televisión pero en menor medida.

Para finalizar, vemos como a pesar de ser blogs de la misma tipología, podemos encontrarnos con algunas diferencias y similitudes de contenidos y recursos permitiendo así darle un enfoque diferente al blog. Los profesores de los blogs se ciñen siempre a los contenidos destinados para cada curso por ello, hemos visto que suelen coincidir a la hora de presentarnos el contenido. Sin embargo, con lo que respecta a los recursos ofrecidos podemos observar que cada uno nos lo presenta de diversas maneras pudiendo comprobar la variedad que existe y como se puede trabajar la Historia de diferentes maneras.

\section{Conclusiones}

La investigación que se ha llevado a cabo, ha tenido como objetivos analizar los contenidos de cinco blogs educativos que tratan sobre temas de índole histórica y por otro lado, comparar estos contenidos con los que ofrecen cada uno de ellos viendo así las diversas maneras en las que se puede enfocar un blog educativo y que no en todos ellos se incluyen los mismos contenidos y recursos. Por consiguiente, hemos comprobado como los nuevos entornos digitales a través de los blogs se nos presentan como una manera alternativa e innovadora de la enseñanza, más en nuestro caso el de la Historia.

Por otro lado, podemos dar respuesta a las cuestiones planteadas al inicio de la investigación. Mediante la investigación, hemos comprobado que los contenidos que nos presentan cada uno de los blogs sí son adecuados para la enseñanza de la Historia, haciendo que se facilite la visión que tiene el alumnado sobre ella y proporcionándole al profesorado una herramienta más para usar en clase, ya que los contenidos que nos ofrecen se adaptan adecuadamente a las exigencias del currículum para cada curso. 
Por otro lado, podemos afirmar que los blogs educativos sí se pueden utilizar como una herramienta para la enseñanza, ya que cada profesor los elabora en relación a las necesidades que tiene su clase y le puede dar una gran cantidad de usos no solo como un sustituto del libro, sino como un espacio de encuentro para subir materiales complementarios, subir las actividades de clase y presentaciones, entre otros usos complementarios de la enseñanza.

Si nos centramos en los capítulos que forman esta investigación, en el primer capítulo hemos comprobado todos los contenidos que se nos presentan en los cinco blogs que han sido analizados. Por ello, podemos mencionar que gracias al análisis de corte cualitativo que hemos emprendido hemos tenido la posibilidad de comprobar la multitud de contenidos de índole histórica que nos ofrecen los blogs seleccionados, además de haber podido observar cómo cada blog selecciona los contenidos a presentar y su grado de profundización, existiendo grandes diferencias a la hora de ofrecer los contenidos de un blog a otro así como, la manera de presentar los contenidos, permitiéndonos comprobar la gran diversidad de recursos que nos proporciona un blog para poder impartir los conocimientos. Por último se ha realizado una comparación de los contenidos y de la manera en la que estos se presentan, pudiendo de esta manera constatar que muchos de los blogs coinciden en ello y destacando las diferencias que hay entre ellos.

\section{Referencias}

Almerich, G., Suárez-Rodríguez, J.M., Belloch, C. \& Bo, R.M. (2011). Las necesidades formativas del profesorado en TIC: Perfiles formativos y elementos de complejidad. Relieve, 17(2), 1-28. Recuperado de https://www.uv.es/RELIEVE/v17n2/RELIEVEv17n2 1.pdf

Aznar Cuadrado, V. y Soto Carballo, J. (2010). Análisis de las aportaciones de los blogs educativos al logro de la competencia digital. Revista de Investigación en Educación, (7), 83-90. Recuperado de http://reined.webs.uvigo.es/ojs/index.php/reined/article/viewFile/81/70

Benito Gómez, M. (2008). Educar en comunidad: tendencias educativas en el nuevo entorno digital. Bit, (167), 37-45. Recuperado de https://www.coit.es/archivo-bit/febrero-marzo-2008/ educar-en-comunidad-tendencias-educativas-en-el-nuevo-entorno-digital

Bisquerra Alzina, R. (Coord.). (2014). Metodología de la investigación educativa. Madrid: La Muralla S.A.

Checa García, F. (2014). Usos específicos de los blogs como herramienta educativa. Nómadas. Revista Crítica de Ciencia Sociales y Jurídicas, 42(2), 105-113. Recuperado de https://revistas.ucm.es/index.php/NOMA/article/view/48773/45520 
Domínguez, R. (2012). Las TIC en la formación del profesorado. Revista científica electrónica de Educación y Comunicación en la Sociedad del Conocimiento, 1(12), 168-179. Recuperado de https://dialnet.unirioja.es/servlet/articulo?codigo=3973273

Fernández Chamorro, V. y Pérez Jiménez, S. (2015). Los blogs en la educación. Un ejemplo práctico. Revista Tecnología, Ciencia y Educación, (2), 30-38. Recuperado de http://tecnologia-ciencia-educacion.com/index.php/TCE/article/view/50

Martín Sánchez, M. A. y Alonso Díaz, L. (2009). Los blogs y su utilidad en la enseñanza de la Historia de la educación. En M. R. Berruezo Albéniz y S. Conejero Lópezz (Coords.) El largo camino hacia una educación inclusiva. La educación especial y social del siglo XIX a nuestros días: XV Coloquio de Historia de la Educación (pp. 737-744). Pamplona: Universidad Pública de Navarra. Recuperado de https://dialnet.unirioja.es/servlet/articulo?codigo $=2964358$

Molina Alventosa, P., Valenciano Valcárcel, J. y Valecia-Peris, A. (2015). Los blogs como entornos virtuales de enseñanza y aprendizaje de Educación Superior. Revista Complutense de Educación, 26(Núm, Especial), 15-31. Recuperado de http://revistas.ucm.es/index.php/RCED/article/view/43791/45929

Roldán Paz, L. (2016). Un ejemplo de innovación educativa en Historia: la utilización de blogs como estrategia de aprendizaje y evaluación. En J. A. Sánchez López y A. R. Fernández Paradas (Coords.) Del individuo al aprendizaje colaborativo (I): la historia y la historia del arte ante los retos de la innovación educativa (pp. 43-54). Antequera, Málaga: Exlibric

Sánchez Agustí, M., González Gallego, I., Revilla, D. M. y Martínez Rodríguez, R. (2015). Entornos de aprendizaje digital para la enseñanza de la historia reciente en España y Chile. Presentación de un proyecto de intervención en secundaria. Andamio, 1(3), 31-48. Recuperado de http://andamio.dynamiclab.cl/index.php/andamio/article/view/25

Sánchez García, J. M. y Toledo Morales, P. (2015). Aproximación al uso de recursos educativos abiertos para Ciencias Sociales en Educación Secundaria y Bachillerato. Prismasocial Revista de Investigación Social, (15), 222-253. Recuperado de http://www.isdfundacion.org/publicaciones/revista/numeros/15/secciones/tematica/t 07 recursos-cienciassociales.html

Sobrino López, D. (2009). Implantación de dos blogs en Geografía e Historia. De “ccss2esoonline” a "senderoshistoria". Clío: History and History Teaching, (35), 1-10. Recuperado de http://clio.rediris.es/n35/blogs.pdf 
Sobrino López, D. (2013). El trabajo con blogs en Ciencias Sociales, Geografía e Historia. Clio: History and History Teaching, (39), 1-49. Recuperado de http://clio.rediris.es/n39/articulos/Sobrinoclio39.pdf

Tamayo Rodríguez, Y. y Ruíz Mulet, A. (2016). Los blogs educativos como recurso didáctico en el proceso de enseñanza-aprendizaje de la historia. Boletín Redipe, 5(9), 73-81. Recuperado de https://revista.redipe.org/index.php/1/article/view/111

Vicent, N. e Ibáñez Á. (2012). El uso de las nuevas tecnologías y el patrimonio en el ámbito escolar. Aula de Innovación Educativa, (208), 22-27. Recupera de https://s3.amazonaws.com/ academia.edu.documents/33045384/2012 Aula Vicent Ibanez.pdf?AWSAccessKey $\underline{\text { Id}=A K I A I W O W Y Y G Z 2 Y 53 U L 3 A \& E x p i r e s=1519319346 \& \text { Signature }=W C x w Q h 1 T}$ mDHK9OcSJK\%2BUU58iuwg\%3D\&response-content-disposition=inline\%3B\%20filename\%3DEl uso de las nuevas tecnologias y el pa.pdf 